\title{
On the Lehmer constant of finite cyclic groups
}

\author{
by \\ Norbert Kaiblinger (Wien)
}

1. Introduction. Let $n$ be a positive integer. Given a polynomial with integer coefficients, $f \in \mathbb{Z}[x]$, denote by $\mathrm{m}_{n}(f)$ its logarithmic Mahler measure over $\mathbb{Z} / n \mathbb{Z}$, defined by

$$
\mathrm{m}_{n}(f)=\frac{1}{n} \sum_{k=0}^{n-1} \log \left|f\left(e^{2 \pi i k / n}\right)\right| .
$$

By $\lambda_{n}>0$ we denote the Lehmer constant of $\mathbb{Z} / n \mathbb{Z}$,

$$
\lambda_{n}=\min _{\substack{f \in \mathbb{Z}[x] \\ \mathbf{m}_{n}(f)>0}} \mathrm{~m}_{n}(f)
$$

(see [11]). We notice later that the minimum is indeed attained, and that it is the same if $\operatorname{deg} f \leq n-1$ is assumed. Lind [1] has given an upper bound for $\lambda_{n}$ (see below) and he obtained the values

$$
\lambda_{1}=\log 2, \quad \lambda_{2}=\frac{1}{2} \log 3, \quad \lambda_{4}=\frac{1}{4} \log 3, \quad \lambda_{n}=\frac{1}{n} \log 2 \quad \text { for all odd } n .
$$

We sharpen his result, complement it by a lower bound, and obtain the value of $\lambda_{n}$ for all $n$ except for multiples of 420 . The main result is formulated in Section 2 and proved in Section 3 .

2. Main result. For a positive integer $n$, let $\left\{\begin{array}{c}\rho(n) \\ \rho^{\prime}(n)\end{array}\right\}$ denote the smallest $\left\{\begin{array}{c}\text { prime number } \\ \text { positive integer }\end{array}\right\}$ that does not divide $n$. We write $p^{k} \| n$ when $p^{k}$ is a principal divisor of $n$, that is, if $p$ is a prime and $k$ is a positive integer such

2010 Mathematics Subject Classification: Primary 11R09; Secondary 11B83, 11C08, $11 \mathrm{~T} 22$.

Key words and phrases: Lind's Lehmer constants, logarithmic Mahler measure, finite cyclic group, cyclotomic polynomial. 
that $p^{k} \mid n$ and $p^{k+1} \nmid n$. Let

$$
\rho^{\prime \prime}(n)=\min \left(\min _{p \nmid n} p, \min _{p^{k} \| n} p^{p^{k}}\right)=\min \left(\rho(n), \min _{p^{k} \| n} p^{p^{k}}\right) .
$$

Lind proved that $\lambda_{n} \leq n^{-1} \log \rho(n)$ for all $n$. Extending his result we obtain the following theorem, our main result.

TheOREM 1. The Lehmer constant of $\mathbb{Z} / n \mathbb{Z}$ is of the form $\lambda_{n}=$ $n^{-1} \log \Lambda_{n}$, with an integer $\Lambda_{n} \geq 2$ not dividing $n$ and in the range

$$
\rho^{\prime}(n) \leq \Lambda_{n} \leq \rho^{\prime \prime}(n) .
$$

For all $n=1, \ldots, 419(\bmod 420)$, we have $\Lambda_{n}=\rho^{\prime}(n)=\rho^{\prime \prime}(n)$.

EXAmple 1. Example new values are $\lambda_{6}=\frac{1}{6} \log 4, \lambda_{8}=\frac{1}{8} \log 3$, or more generally,

$$
\lambda_{n}=\left\{\begin{array}{l}
\frac{1}{n} \log 3 \quad \text { if, and only if, } n=2 k \text { with } 3 \nmid k, \\
\frac{1}{n} \log 4 \quad \text { if, and only if, } n=6 k \text { with odd } k .
\end{array}\right.
$$

REMARK 1. (i) Theorem 1 yields the exact value of $\lambda_{n}$ when $\rho^{\prime}(n)=$ $\rho(n)$, or more generally, when $\rho^{\prime}(n)=\rho^{\prime \prime}(n)$. Thus it also includes certain multiples of 420 . For example, let $n=6 \cdot k \cdot 420$ with $11 \nmid k$. Then $\rho^{\prime}(n)=$ $\rho(n)=11$ and thus $\lambda_{n}=n^{-1} \log 11$.

(ii) By Theorem 1 the known upper bound $\lambda_{n} \leq n^{-1} \log \rho(n)$ is sharpened strictly for all $n=6(\bmod 12)$, where it yields the exact value for $\lambda_{n}$, and also for certain multiples of 420 . For example, let $n=11 \cdot 13 \cdot 420$. Then the theorem implies $\lambda_{n}=n^{-1} \log \Lambda_{n}$ with $\Lambda_{n} \in\{8,9,16\}$, while $\rho(n)=17$.

Open Question. Determine $\lambda_{n}=n^{-1} \log \Lambda_{n}$ for $n=420$. By Theorem 1 (i) we have $\Lambda_{420} \in\{8,9,11\}$.

3. Proof of Theorem 1. We have, for $f \in \mathbb{Z}[x]$,

$$
\mathrm{m}_{n}(f)=\frac{1}{n} \log \left|\Delta_{n}(f)\right| \quad \text { with } \quad \Delta_{n}(f)=\prod_{k=0}^{n-1} f\left(e^{2 \pi i k / n}\right) .
$$

The number $\Delta_{n}(f)$ is always an integer, and there is an elementary way to see that. To this end we recall the determinantal relation of [13, readily extended here to $f$ of arbitrary degree. If $\operatorname{deg} f \leq n-1$, write $f(x)=$ $a_{0}+a_{1} x+\cdots+a_{n-1} x^{n-1}$, with zero coefficients where necessary. If a polynomial of higher degree is given, with coefficients $a_{0}^{\prime}, a_{1}^{\prime}, \ldots$, replace it first with $f$ as above by defining $a_{k}=\sum_{l=k(\bmod n)} a_{l}^{\prime}$. Let $C_{a}$ denote the $n \times n$ integer circulant matrix with first row $a=\left(a_{0}, \ldots, a_{n-1}\right)$. Then $\operatorname{det} C_{a}=\prod_{k=0}^{n-1} f\left(e^{2 \pi i k / n}\right)$ and this implies

$$
\Delta_{n}(f)=\operatorname{det} C_{a} .
$$


Hence, $\Delta_{n}(f)$ is indeed an integer. Observe that expressing $\mathrm{m}_{n}(f)$ in terms of the integer $\Delta_{n}(f)$ justifies the definition of the Lehmer constant $\lambda_{n}$ as a minimum, not just an infimum. We will also use the expression of $\Delta_{n}(f)$ as a resultant, like for example in [2, 5, 11]. Indeed, since $\operatorname{Res}\left(x^{n}-1, f(x)\right)=$ $\prod_{k=0}^{n-1} f\left(e^{2 \pi i k / n}\right)$, we have

$$
\Delta_{n}(f)=\operatorname{Res}\left(x^{n}-1, f(x)\right) .
$$

The more commonly used expression $\operatorname{Res}\left(f(x), x^{n}-1\right)$, with interchanged arguments, works as well, as long as only absolute values are considered. Indeed, the sign of the determinant in (2) or of the resultant in (3) is irrelevant for $\mathrm{m}_{n}(f)$. We remark that the opposite sign is obtained for the polynomial

$$
f^{\vee}(x)=-x^{n-1} f(1 / x),
$$

with coefficient sequence $\left(-a_{n-1},-a_{n-2}, \ldots,-a_{0}\right)$, the negative of the usual reciprocal polynomial.

REMARK 2. (i) Lehmer and Pierce [10, 13, investigated the sequences $\left\{\Delta_{1}(f), \Delta_{2}(f), \ldots\right\}$ for $f \in \mathbb{Z}[x]$. For example, the polynomial $f(x)=2-x$ yields $\Delta_{n}(f)=2^{n}-1$, the Mersenne numbers; we refer to [6, 7, 8, 9]. For Lehmer's problem, formulated in [10], we refer to [3, 14] and the spectacular solution for odd coefficients in [2]. Lind's Lehmer constants $\lambda_{n}$ relate to the family $\left\{\Delta_{n}(f): f \in \mathbb{Z}[x]\right\}$ for fixed $n$.

(ii) Our approach highlights and makes use of the fact that finding possible (or minimal) values of the logarithmic Mahler measure over $\mathbb{Z} / n \mathbb{Z}$ is equivalent to finding possible (or minimal) values of integer circular determinants, an open problem attributed to Taussky-Todd [12].

Call $f \in \mathbb{Z}[x]$ cyclotomic if all its zeros lie on the complex unit circle. As a preliminary observation we determine, for all $n$, the exact value of a cyclotomic variant of Lind's Lehmer constants.

Lemma 1. For cyclotomic polynomials $f \in \mathbb{Z}[x]$, the minimal possible value of $\mathrm{m}_{n}(f)>0$ is determined by

$$
\min _{\substack{f \in \mathbb{Z}[x] \text { cyclotomic } \\ \mathbf{m}_{n}(f)>0}} \mathrm{~m}_{n}(f)=n^{-1} \log \rho^{\prime \prime}(n) .
$$

Proof. First, Kronecker's theorem implies that any cyclotomic polynomial $f \in \mathbb{Z}[x]$ is the product of some of $\Phi_{1}, \Phi_{2}, \ldots$ and a constant, if necessary; here $\Phi_{m} \in \mathbb{Z}[x]$ denotes the $m$ th cyclotomic polynomial, i.e., the monic polynomial whose zeros are the primitive $m$ th roots of unity. Since always

$$
\Delta_{n}\left(f_{1} f_{2}\right)=\Delta_{n}\left(f_{1}\right) \Delta_{n}\left(f_{2}\right),
$$

and consequently, $\mathrm{m}_{n}\left(f_{1} f_{2}\right) \leq \mathrm{m}_{n}\left(f_{1}\right)+\mathrm{m}_{n}\left(f_{2}\right)$, we thus obtain

$$
\min _{\substack{f \in \mathbb{Z}[x] \text { cyclotomic } \\ \mathbf{m}_{n}(f)>0}} \mathrm{~m}_{n}(f)=\min _{\substack{m=1,2, \ldots \\ \mathbf{m}_{n}\left(\Phi_{m}\right)>0}} \mathrm{~m}_{n}\left(\Phi_{m}\right) .
$$


Let $\varphi(n)$ denote Euler's totient of $n$. We point out that

$$
\begin{aligned}
& \Delta_{n}\left(\Phi_{m}\right)=\operatorname{Res}\left(x^{n}-1, \Phi_{m}(x)\right) \\
& = \begin{cases}0 & \text { if } m \mid n, \\
1 & \text { if at least two distinct primes divide } m / \operatorname{gcd}(m, n), \\
p^{\varphi(q)} & \text { if } m / \operatorname{gcd}(m, n) \text { is the power of a prime } p \nmid n \\
& \text { - here we write } \operatorname{gcd}(m, n)=q, \\
p^{\varphi(q) p^{k}} & \text { if } m / \operatorname{gcd}(m, n) \text { is the power of a prime } p \mid n \\
& \text { - here we factorize } \operatorname{gcd}(m, n)=p^{k} q \text { with } p^{k} \| n .\end{cases}
\end{aligned}
$$

We remark that by our approach no negative sign is needed here, for any $m, n$. This formula is obtained from [1, proof of Theorem 2], where it is used for a short proof of the formula for $\operatorname{Res}\left(\Phi_{m_{1}}(x), \Phi_{m_{2}}(x)\right)$; secondly, since

$$
\operatorname{Res}\left(x^{n}-1, \Phi_{m}(x)\right)=\operatorname{Res}\left(\Phi_{1}\left(x^{n}\right), \Phi_{m}(x)\right),
$$

the formula (7) also follows from applying [4, Proposition 14]; a third, convenient and direct source is [5, Theorem 3].

Notice that (7) implies, for any $n, m$, that in particular

$$
\Delta_{n}\left(\Phi_{m}\right)=0,1, \quad \text { or } \quad \Delta_{n}\left(\Phi_{m}\right) \geq \min \left(\min _{p \nmid n} p, \min _{p^{k} \| n} p^{p^{k}}\right)=\rho^{\prime \prime}(n) .
$$

Since (7) also yields

$$
\begin{aligned}
& \Delta_{n}\left(\Phi_{p}\right)=p \quad \text { for } p \nmid n, \\
& \Delta_{n}\left(\Phi_{p^{k+1}}\right)=p^{p^{k}} \quad \text { for } p^{k} \| n,
\end{aligned}
$$

we conclude that the inequality in $(9)$ is sharp, that is,

$$
\min _{\substack{m=1,2, \ldots \\ \Delta_{n}\left(\Phi_{m}\right) \geq 2}} \Delta_{n}\left(\Phi_{m}\right)=\rho^{\prime \prime}(n)
$$

Finally, since $\mathrm{m}_{n}\left(\Phi_{m}\right)=n^{-1} \log \Delta_{n}\left(\Phi_{m}\right)$, the statement of the lemma follows by combining (6) and (11).

Lemma 2. Let $n$ satisfy $n \neq 6(\bmod 12)$ and $n \neq 0(\bmod 420)$. Then $\rho(n)=\rho^{\prime}(n)$, that is, the least non-divisor of $n$ is a prime (and not a prime power).

REMARK 3. The example given in Remark 1(i) shows that the implication of Lemma 2 cannot be reversed.

Proof of Lemma 2. Suppose that $n \neq 6(\bmod 12)$ and $\rho^{\prime}(n)<\rho(n)$; we verify that this implies $420 \mid n$. First, if $6 \nmid n$, then either $\rho^{\prime}(n)=\rho(n)=2$ or $\rho^{\prime}(n)=\rho(n)=3$. This contradicts the assumption $\rho^{\prime}(n)<\rho(n)$. Hence, we have $n=6 k$ for some $k$. The case of $k$ odd is excluded by the assumption 
$n \neq 6(\bmod 12)$, so we obtain $k$ even. In other words, $n=12 k^{\prime}$ for some $k^{\prime}$. If $5 \nmid k^{\prime}$, then we have $\rho^{\prime}(n)=\rho(n)=5$, in contradiction to the assumption $\rho^{\prime}(n)<\rho(n)$. Therefore, we have $n=60 k^{\prime \prime}$ for some $k^{\prime \prime}$. Finally, if $7 \nmid k^{\prime \prime}$, then $\rho^{\prime}(n)=\rho(n)=7$, again contrary to $\rho^{\prime}(n)<\rho(n)$. Thus we conclude that $n=420 k^{\prime \prime \prime}$ for some $k^{\prime \prime \prime}$.

Proof of Theorem 1 .

STEP I: First notice that indeed $\lambda_{n}=n^{-1} \log \Lambda_{n}$ for an integer $\Lambda_{n} \geq 2$; in fact,

$$
\Lambda_{n}=\min _{\substack{f \in \mathbb{Z}[x] \\\left|\Delta_{n}(f)\right| \geq 2}}\left|\Delta_{n}(f)\right| .
$$

Therefore, $\Lambda_{n}=\left|\Delta_{n}\left(f_{0}\right)\right|$ for some $f_{0} \in \mathbb{Z}[x]$ with $\operatorname{deg} f_{0}=n-1$. Upon replacing $f_{0}$ with $f_{0}^{\vee}$ defined above, if necessary, we can assume that $\Lambda_{n}=$ $\Delta_{n}\left(f_{0}\right)$.

STEP II: We show that $\Lambda_{n} \nmid n$. Suppose that $\Lambda_{n}$ divides $n$. Then there exists a prime $p$ dividing both $\Lambda_{n}$ and $n$. Let $p^{m} \| \Lambda_{n}$ and $p^{k} \| n$. Since $\Lambda_{n} \mid n$ we notice that $m \leq k$. On the other hand, let $C_{a}$ be the $n \times n$ integer circulant matrix whose first row consists of the coefficients of $f_{0}$, so that

$$
\Lambda_{n}=\Delta_{n}\left(f_{0}\right)=\operatorname{det} C_{a} .
$$

Then we have $p^{k} \| n$ and $p^{m} \| \operatorname{det} C_{a}$, and a result by Newman [12, Theorem 2] thus implies that $m \geq k+1$, so we obtain a contradiction.

STEP III: The previous step implies that the positive integer $\Lambda_{n}$ does not divide $n$. By definition, $\rho^{\prime}(n)$ is the smallest number with this property. We thus obtain the lower bound $\rho^{\prime}(n) \leq \Lambda_{n}$.

STEP IV: The upper bound $\Lambda_{n} \leq \rho^{\prime \prime}(n)$ is a consequence of Lemma 1 .

Step V: Suppose that $n=6(\bmod 12)$. Then $2 \mid n$ and $3 \mid n$, while $4 \nmid n$. Hence, $\rho^{\prime}(n)=4$. On the other hand,

$$
\min _{p^{k} \| n} p^{p^{k}}=2^{2^{1}}=4
$$

and thus $\rho^{\prime \prime}(n)=4$; notice that $\rho(n) \geq 5$. Therefore in Theorem 1 the lower and upper bound coincide, and we obtain $\Lambda_{n}=\rho^{\prime}(n)=\rho^{\prime \prime}(n)=4$.

STEP VI: Suppose that $n \neq 6(\bmod 12)$ and $n \neq 0(\bmod 420)$. By Lemma 2 these conditions on $n$ imply that $\rho(n)=\rho^{\prime}(n)$. Since always $\rho^{\prime}(n) \leq$ $\rho^{\prime \prime}(n) \leq \rho(n)$, we conclude that $\rho^{\prime}(n)=\rho^{\prime \prime}(n)$. Thus the lower and upper bound in Theorem 1 coincide and we obtain $\Lambda_{n}=\rho^{\prime}(n)=\rho^{\prime \prime}(n)$.

Acknowledgments. This research was supported by the Austrian Science Fund FWF grant P 21339. 


\section{References}

[1] T. M. Apostol, Resultants of cyclotomic polynomials, Proc. Amer. Math. Soc. 24 (1970), 457-462.

[2] P. Borwein, E. Dobrowolski, and M. J. Mossinghoff, Lehmer's problem for polynomials with odd coefficients, Ann. of Math. 166 (2007), 347-366.

[3] D. W. Boyd, Mahler's measure and special values of L-functions, Experiment. Math. 7 (1998), 37-82.

[4] C. C. Cheng, J. H. McKay, and S. S.-S. Wang, Resultants of cyclotomic polynomials, Proc. Amer. Math. Soc. 123 (1995), 1053-1059.

[5] J. E. Cremona, Unimodular integer circulants, Math. Comp. 77 (2008), 1639-1652.

[6] M. Einsiedler, G. Everest, and T. Ward, Primes in sequences associated to polynomials (after Lehmer), LMS J. Comput. Math. 3 (2000), 125-139.

[7] G. Everest, P. Rogers, and T. Ward, A higher-rank Mersenne problem, in: Algorithmic Number Theory, W. L. Mang, C. Fieker, and D. R. Kohel (eds.), Springer, Berlin, 2002, 95-107.

[8] A. Flatters, Primitive divisors of some Lehmer-Pierce sequences, J. Number Theory 129 (2009), 209-219.

[9] C. J. Hillar and L. Levine, Polynomial recurrences and cyclic resultants, Proc. Amer. Math. Soc. 135 (2007), 1607-1618.

[10] D. H. Lehmer, Factorization of certain cyclotomic functions, Ann. of Math. 34 (1933), 461-479.

[11] D. Lind, Lehmer's problem for compact abelian groups, Proc. Amer. Math. Soc. 133 (2005), 1411-1416.

[12] M. Newman, On a problem suggested by Olga Taussky-Todd, Illinois J. Math. 24 (1980), 156-158.

[13] T. A. Pierce, The numerical factors of the arithmetic forms $\prod_{i=1}^{n}\left(1 \pm \alpha_{i}^{m}\right)$, Ann. of Math. 18 (1916), 53-64.

[14] C. Smyth, The Mahler measure of algebraic numbers: a survey, in: Number Theory and Polynomials, J. McKee and C. Smyth (eds.), Cambridge Univ. Press, 2008, 322-349.

Norbert Kaiblinger

Faculty of Mathematics

University of Vienna

Nordbergstraße 15

1090 Wien, Austria

E-mail: norbert.kaiblinger@univie.ac.at

Received on 27.4.2009

and in revised form on 28.8.2009

(6011) 